\title{
Electro-Quasistatic Simulations in Bio-Systems Engineering and Medical Engineering
}

\author{
U. van Rienen ${ }^{1}$, J. Flehr ${ }^{1}$, U. Schreiber ${ }^{1}$, S. Schulze ${ }^{1}$, U. Gimsa ${ }^{3}$, W. Baumann ${ }^{2}$, D. G. Weiss ${ }^{2}$, J. Gimsa ${ }^{2}$, R. Benecke ${ }^{3}$, \\ and H.-W. Pau ${ }^{3}$ \\ ${ }^{1}$ Rostock University, Faculty of Computer Science and Electrical Engineering, Albert-Einstein-Str. 2, \\ 18051 Rostock, Germany \\ ${ }^{2}$ Rostock University, Faculty of Mathematics and Natural Sciences, Albert-Einstein-Str. 3, 18051 Rostock, Germany \\ ${ }^{3}$ Rostock University, Faculty of Medicine, PF 1008 88, 18055 Rostock, Germany
}

\begin{abstract}
Slowly varying electromagnetic fields play a key role in various applications in bio-systems and medical engineering. Examples are the electric activity of neurons on neurochips used as biosensors, the stimulating electric fields of implanted electrodes used for deep brain stimulation in patients with Morbus Parkinson and the stimulation of the auditory nerves in deaf patients, respectively. In order to simulate the neuronal activity on a chip it is necessary to couple Maxwell's and Hodgkin-Huxley's equations. First numerical results for a neuron coupling to a single electrode are presented. They show a promising qualitative agreement with the experimentally recorded signals. Further, simulations are presented on electrodes for deep brain stimulation in animal experiments where the question of electrode ageing and energy deposition in the surrounding tissue are of major interest. As a last example, electric simulations for a simple cochlea model are presented comparing the field in the skull bones for different electrode types and stimulations in different positions.
\end{abstract}

\section{Introduction}

There is a great number of applications in bio-systems engineering or medical engineering where slowly varying electromagnetic fields play a key role. Some examples will be treated in this paper as there are: the electric activity of neurons on neurochips used as sensors in various application fields and the stimulating electric fields of implanted electrodes used for deep brain stimulation in patients with Morbus Parkinson and stimulation of the auditory nerves in deaf patients, respectively. Even though these processes happen in milliseconds they are quasistationary as the typical dimensions, e.g. of the electrodes in the inner ear are small compared to the typical wavelength of the stimulation frequency. Quasistationary electromagnetic fields subdivide

Correspondence to: U. van Rienen

(ursula.van-rienen@uni-rostock.de) in the so-called electro- and magneto-quasistatics depending on whether the electric or magnetic field clearly dominates. Some quantitative criteria are given in the paper. The problems studied here may be classified as electro-quasistatic. The analytical equations as well as their numerical counterpart are derived from Maxwell's equations. As numerical method, the Finite Integration Technique is used throughout this paper. In order to simulate the neuronal activity on a chip it is necessary to couple Maxwell's equations to HodgkinHuxley's equations which describe the neuronal activity. For explanation, a brief summary of some important facts from neurophysiology will be given together with the HodgkinHuxley equations. A first step towards a numerical model is presented which allows computing the neuronal signal at the electrodes of a neurochip. Results for a simple set-up with a single neuron and one electrode show a promising qualitative agreement with the signals seen in experiments. As the determining factors for a special signal pattern are still unknown the numerical model will be used in future to study the functional relations between neurochip parameters and signal patterns.

For implanted electrodes, the electric fields and currents in the close neighborhood are strongly depending on the electrode geometry and their stimulation pattern. Besides the nerve-stimulation effect, also unwanted side effects play an important role in the clinical practice. This problem is addressed in simulations of electrodes for deep brain stimulation in animals. In these applications, electrode ageing and energy deposition in the surrounding tissue are of main interest. The simulations allowed for geometrical qualification criteria for these electrodes.

Regarding cochlear implants used for direct stimulation of the auditory nerves in deaf patients one practical problem is the unwanted stimulation of the facial nerves often seen in patients with otosclerosis. For a simple cochlear model some simulations are presented comparing the field in the bones for different electrode types and stimulation in different positions. 


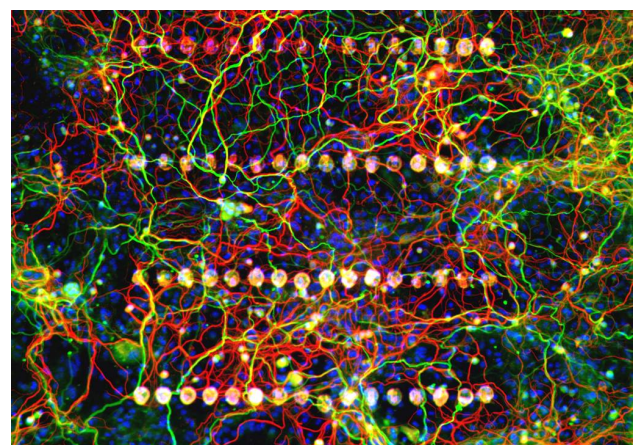

Fig. 1. Neuronal network culture on a 64-electrode array. Frontal cortex 39 div, green: NF 200, red: MAP2, blue: nuclei. Microelectrode array: G. W. Gross, CNNS, Univ. North Texas. Photo: S. Stüwe.

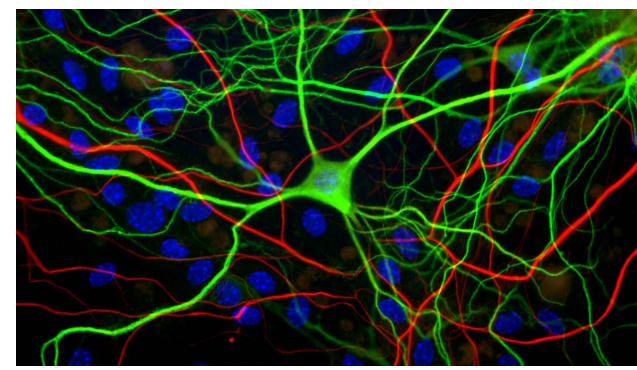

Fig. 2. Neuronal network culture. Frontal cortex 28 days in vitro dendrites stained with MAP2 (green) and axons stained with $200 \mathrm{kD}$ MAP3 like antibody (red; courtesy of Walter Steffen), nuclei blue. Photo: S. Stüwe.

\section{Neurochips}

\subsection{Some basic facts about neurons}

All processes in humans or animals rely on information provided by other parts of the body. The information transport is provided by the neuronal system which itself is based on neurons. Neurons transport information by electric impulses, also called nerve impulses. Neurons in the human body can live for about 100 years. The maximum of $10^{11}$ nerve cells (neurons) is reached soon after birth, from then on there is a loss during maturation of up to $90 \%$ followed by small daily losses.

Each neuron consists of three main parts: 1. the soma or cell body, 2. the axon, 3. the dendrites as shown in Fig. 2. Typically, a neuron has only one axon but there are also neurons in the central nerve system without any. The axon may be up to one meter long. Such a long axon is called nerve fibre. There exist various diameters of an axon - the larger the diameter, the faster the nerve impulse. Finally, the neural axon may have a myelin sheath (oligodendrocyte and Schwann cell covering in the central and peripheral nervous system, respectively) or not. The dendrites are receiving up to 200000 synapses.

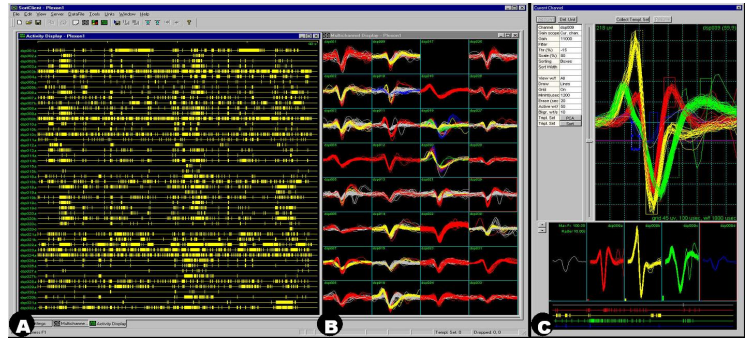

Fig. 3. Multi-channel recording of the activity on a neurochip. (A): Activity (bursts), (B): multi-channel display, (C): single channel display.

The neural information processing acts as follows: The synapses ending at the dendrites and soma transport the information from other neurons as positive or negative charge. The charges are added up in space and time - spatially several weak signals are converted into one strong signal, temporally a fast series of weak impulses from one and the same source is converted in one strong signal only. The total signal is transferred to the soma. If the total signal is greater than the axon hillock's threshold an initial signal is guided along the axon - the axon "fires". The strength of the initial signal is constant, independently of the threshold size. The initial signal reaches all terminal buttons.

In order to describe the neuronal activity it is necessary to introduce the resting potential and the action potential: The so-called resting potential of an axon at rest can be measured with the classical setup of an oscilloscope, an amplifier, a container with a reference electrode and a microelectrode pinched inside the axon under study. A voltage of $60-90 \mathrm{mV}$ will be measured with the inside negative relative to the outside. This voltage is denoted as resting potential (even though it is a potential difference) and the convention is that the sign of the inside is taken, i.e. the resting potential takes values of -60 to $-90 \mathrm{mV}$. After stimulation of the axon in this measurement setup the electric impulses (spikes) run along the axon. When a pulse passes the measurement device the resting potential first decreases to zero, then the inner axon gets positive and the voltage increases rapidly to +20 to $+40 \mathrm{mV}$. Therein the maximal value depends on the type of neuron. Thus the total voltage change amounts to about $100 \mathrm{mV}$. These $100 \mathrm{mV}$ impulses are called action potentials. Only $0.5-1 \mathrm{~ms}$ later the resting potential is established again.

The neuronal activity is an electrochemical process. A key role in that play the concentration difference of cations and anions inside and outside the neuronal membrane as well as the permeability of the membrane with respect to cations and anions. The membrane properties provide the charge separation which leads to the resting potential. Resting potential and action potential are diffusion potentials. Through changes in permeability according to the Goldman-HodgkinKatz equation (see e.g. Keener and Sneyd (2001)) the potassium $\left(\mathrm{K}^{+}\right)$potential dominates in the resting state while the 
sodium $\left(\mathrm{Na}^{+}\right)$potential dominates in the active state; chloride $\left(\mathrm{Cl}^{-}\right)$plays a rather passive role. During the active state voltage-controlled sodium and potassium channels enable a depolarization. In the resting state the membrane is highly permeable for $\mathrm{K}^{+}$-ions, somewhat permeable to $\mathrm{Cl}^{-}$-ions and barely permeable to $\mathrm{Na}^{+}$-ions while it is not permeable to anions. During the action potential the permeability for $\mathrm{Na}^{+}$-ions increases momentarily by a factor of about 100 .

The transmission of the stimulation is different for axons with or without myelin sheath. Without myelin sheath the depolarization continuously propagates along the axon. In the measurement setup the propagation of depolarization is possible in both directions after stimulation in the middle of the axon. In the nervous system it is always only one direction of propagation. The propagation speed depends on the axon diameter, e.g. in cats' pain fibres of $1 \mu \mathrm{m}$ diameter the propagation speed reaches $1 \mathrm{~m} / \mathrm{s}$ while it is $20 \mathrm{~m} / \mathrm{s}$ in the $500 \mu \mathrm{m}$ thick nerve fibres of the giant squid. In axons with myelin sheath the action potential jumps from Ranvier node to Ranvier node. This propagation is much faster why the propagation speed reaches up to $130 \mathrm{~m} / \mathrm{s}$ for axons with 10-20 $\mu \mathrm{m}$ diameter.

The interested reader can find much more information in Keener and Sneyd (2001).

\subsection{Cell Monitoring System with silicon based neurochip}

A Cell Monitoring System with a silicon based neurochip for the measurement of the electrical as well as metabolic activity of neuronal networks has been realized in the Biophysics department at Rostock University (Baumann and Lehmann, 2004). Therewith, metabolic as well as electrical activity can be measured on neuronal networks. Living cells are directly cultured on the neuro-sensorchip (see Figs. 4 and 5). Neuronal cells from dissociated tissue cultures were prepared according to the basic method established by Ransom and Nelson (1977). Neurons were maintained for one week in minimal essential medium (MEM), containing 10\% fetal calf serum and $10 \%$ horse serum. Thereafter cells were fed 3times per week with MEM containing $10 \%$ horse serum. The cultures were maintained at $37^{\circ} \mathrm{C}$ in an atmosphere with $10 \%$ $\mathrm{CO}_{2}$. The network developed spontaneous electrical activity after about 1 week and stabilized after 3 weeks. The culture area on the chip is less than $20 \mathrm{~mm}^{2}$ and has a chamber volume of $10 \mu \mathrm{l}$ in the flow injection system.

On the present neurochip, a palladium multi electrode array (MEA) with $58 \mathrm{Pd}$ electrodes, different types of cell potential field effect transistors (CPFETs), temperature sensors, ion sensitive FETs (ISFETs) and two types of oxygen sensors were integrated. Therewith metabolic and electrical signals can be measured in parallel on neuronal networks, cultured on the sensor chip. As metabolic parameters, the acidification (see Fig. 6) and respiration rate of the cells can be measured. Additionally, the action potential signals of neuronal networks cultured on this CMOS neurosensor microchip can be measured non-invasive due to extracellular coupling between sensor and cell/axon (see Fig. 7).

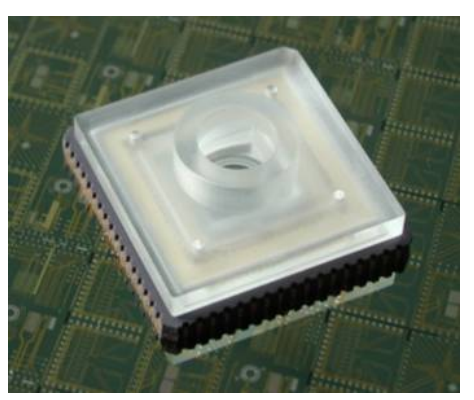

Fig. 4. CMOS sensorchip with CNC encapsulation on standard 68pin PLCC ceramic socket. The cells are cultured directly in the trough on the chip. Bionas $\mathrm{GmbH}$ (Bio).

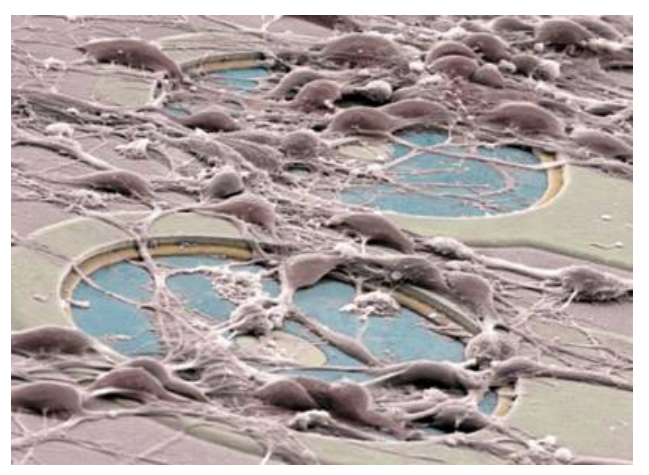

Fig. 5. SEM picture of neuronal network at the beginning of growth (after 3 days) on electrodes. After about 3 weeks the culture area of the chip is completely overgrown by cells. Photo: S. Stüwe.

Optimization of the cell-sensor coupling can be achieved with electrical simulations of this biohybrid system.

\subsection{A mathematical model for neurochips}

\subsubsection{The Hodgkin-Huxley equations}

Cell membranes can be viewed as capacitors since they separate charge. The capacitance of a cell membrane is typically found to be $1.0 \mu \mathrm{F} / \mathrm{cm}^{2}$, corresponding to a relative permittivity $\varepsilon_{r}$ of about 8.5 (Keener and Sneyd, 2001). The simple electrical circuit model shown in Fig. 8 is the basis for much of theoretical electrophysiology. It models the cell membrane as a capacitor in parallel with an ionic current, resulting in the equation

$C_{m} \frac{d V}{d t}+I_{\text {ionic }}(V, t)=0$,

where $V$ denotes the membrane potential, i.e. internal minus external potential $\left(V=V_{i}-V_{e}\right)$.

The first quantitative model of the propagation of an electrical signal along an axon has been developed in 1952 by Hodgkin and Huxley (1952). In many neural cells the sodium current $I_{\mathrm{Na}}$ and the potassium current $I_{\mathrm{K}}$ are the principal ionic currents. The other relatively small ionic currents 


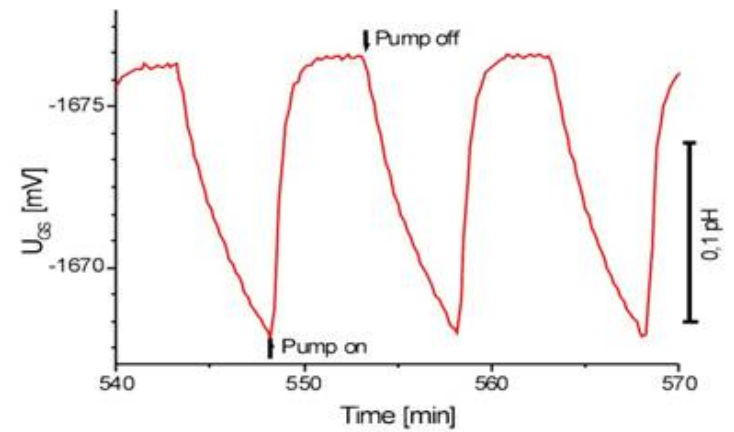

Fig. 6. Cutout from an extracellular acidification measurement of a neuronal network on a silicon sensorchip with ISFETs in a flow through system. The acidification was measured at each pump off cycle. During the pump on cycle the medium was completely exchanged with fresh medium.

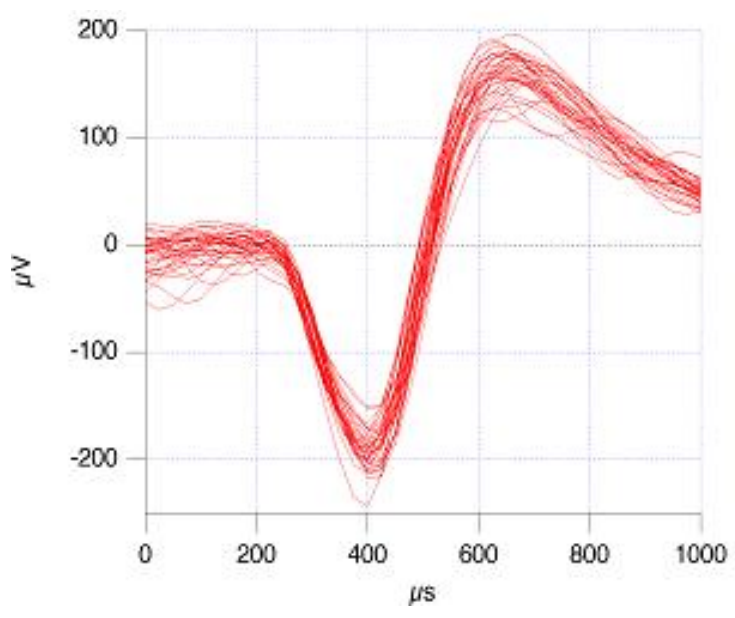

Fig. 7. Extracellular action potentials measured with Pd electrodes on the silicon neurosensor chip.

(primarily the chloride current) are lumped together in the Hodgkin-Huxley theory into one current called the leakage current $I_{l}$ :

$$
I_{\text {ionic }}=I_{\mathrm{Na}}+I_{\mathrm{K}}+I_{l} \text {. }
$$

The individual ionic currents are linearly related to the driving potential via Ohm's law.

Thus, in the Hodgkin-Huxley model the cell membrane is modelled by a first-order ordinary differential equation:

$$
\begin{aligned}
C_{m} \frac{d V}{d t}= & -\left(G_{\mathrm{Na}}(V(t), t)\left(V(t)-E_{\mathrm{Na}}\right)\right. \\
& +G_{\mathrm{K}}(V(t), t)\left(V(t)-E_{\mathrm{K}}\right) \\
& \left.+G_{l}(V(t), t)\left(V(t)-E_{l}\right)\right) \\
= & -\left(\bar{G}_{\mathrm{Na}} m^{3} h\left(V-E_{\mathrm{Na}}\right)+\bar{G}_{\mathrm{K}} n^{4}\left(V-E_{\mathrm{K}}\right)\right. \\
& \left.+G_{l}\left(V-E_{l}\right)\right),
\end{aligned}
$$

where $V$ is the voltage across the membrane, $E_{\mathrm{Na}}, E_{\mathrm{K}}$ and $E_{l}$ are the ionic reversal potential given by Nernst's equation - the equilibrium value, and $G_{\mathrm{Na}}, G_{\mathrm{K}}$ and $G_{l}$ are the ionic

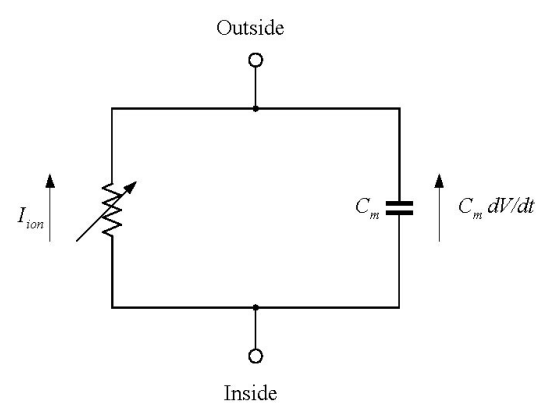

Fig. 8. Simple electrical circuit model of the cell membrane.

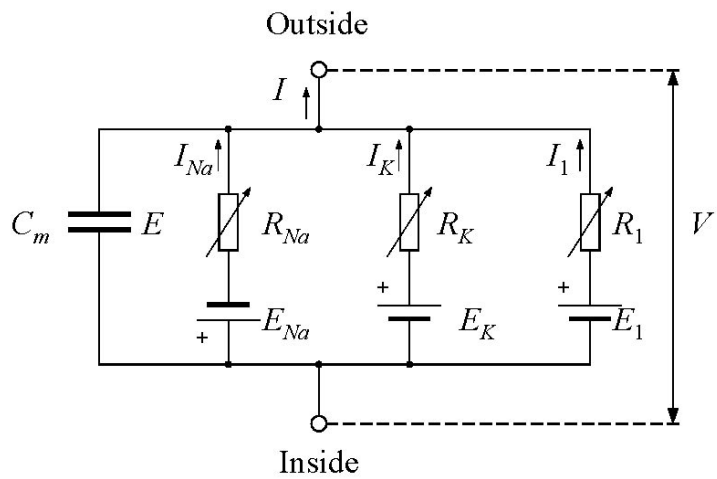

Fig. 9. Electrical circuit model of the cell membrane distinguishing the principal ionic currents.

conductances. The time- and voltage-dependent sodium and potassium conductances $G_{\mathrm{Na}}$ and $G_{\mathrm{K}}$ are depicted in terms of a number of activating or inactivating gating particles multiplied by the maximal conductance $\bar{G}_{\mathrm{Na}}$ and $\bar{G}_{\mathrm{K}}$, respectively. Each gating particle can be either in an open or closed state. In order for the conductance to open, all of these gating particles must be open simultaneously. In detail, the state of $G_{\mathrm{Na}}$ is governed by three activation particles $m$ and one inactivating particle $h$, while the state of $G_{\mathrm{K}}$ is regulated by four activating particles $n$. First order differential equations govern the dynamics of these particles:

$$
\begin{aligned}
& \frac{d m}{d t}=\alpha_{m}(V)(1-m)-\beta_{m}(V) m, \\
& \frac{d h}{d t}=\alpha_{h}(V)(1-h)-\beta_{h}(V) h, \\
& \frac{d n}{d t}=\alpha_{n}(V)(1-n)-\beta_{n}(V) n,
\end{aligned}
$$

with voltage-dependent rate constants $\alpha_{i}, \beta_{i}$ (in units $1 / \mathrm{s}$ ), specifying how many transitions occur between the closed and the open state or vice versa, respectively. These are empirically approximated by some functions (Koch, 1999).

Hence, the electrical circuit model of Fig. 8 can be refined as shown in Fig. 9

Thus, the current across the axon's membrane can be computed by the Hodgkin-Huxley equations. In a setup like on a neurochip, it is of interest to simulate the ensemble of the 
close neighborhood of the neuron including especially the electrodes together with the neuron itself. This means that Maxwell's equations, which describe all macroscopic electromagnetic phenomena, have to be coupled to the HodgkinHuxley equations. It is not necessary to use the full set of Maxwell's equations in its complete generality since the electric phenomena which are of interest can be described by the so-called electro-quasistatic equations. This means that only one ordinary differential equation has to be taken into account instead of the full set of four coupled partial differential equations.

\subsubsection{Maxwell's equations and the EQS model}

Maxwell's equations describe all macroscopic electromagnetic phenomena reflecting the relations between the characteristic quantities of electromagnetic fields, the space- and time-dependent electric and magnetic field strength $\mathbf{E}$ and H. In applications where the wavelength of the studied electromagnetic fields is large compared to the extension of the studied object the wave propagation phenomena may be neglected, i.e. either electric or magnetic fields can be regarded. These "slowly-varying" fields are denoted as electro- and magneto-quasistatic, respectively.

Electro-quasistatics (EQS) gives a reasonable approximation for slowly-varying fields which can be thought to be free of eddy currents while the effects of the displacement current are dominant. This approximation is valid if the problem under study shows a capacitive behavior, i.e. an electrostatic field, for the idealized model of the static limit with frequency $f \rightarrow 0$. The relation

$\tau_{d}=\mu \sigma d^{2} \ll \tau_{r}=\varepsilon / \sigma$

with the diffusion time $\tau_{d}$ and the relaxation time $\tau_{r}$ (unit s), the permeability $\mu$, electrical conductivity $\sigma$, electrical permittivity $\varepsilon$ and a characteristic dimension $d$ of the system under study can be used as a general criterion for EQS. A derivation of estimations about the regime in which the quasistationary approximation is valid can be found in Haus and Melcher (1989), compare also Dirks (1996). For the timeharmonic case the criterion

$|k d| \ll 1 \quad$ with $\quad k=\omega \sqrt{\mu \varepsilon\left(1-i \frac{\sigma}{\omega \varepsilon}\right)}$,

where $k$ is the wave number and $\omega$ is the circular frequency $\omega=2 \pi f$ can be used.

According to the EQS assumptions the electric field $\mathbf{E}$ is free from eddy currents, so the field can be described uniquely by a scalar potential function (shortly potential):

$\mathbf{E}(\mathbf{r}, t)=-\operatorname{grad} \varphi(\mathbf{r}, t)$.

Then, replacing the electric field strength $\mathbf{E}$ in AmpèreMaxwell's law by (9) and after some mathematical manipulations described in van Rienen and Motrescu (2003) the governing partial differential equation for EQS with time invariant, locally constant material is obtained:

$\Delta\left[\underline{\underline{\varepsilon}} \frac{\partial \varphi(\mathbf{r}, t)}{\partial t}+\underline{\underline{\sigma}} \varphi(\mathbf{r}, t)\right]=\operatorname{div} \mathbf{J}_{E}(\mathbf{r}, t)$, where $\underline{\varepsilon}$ and $\underline{\sigma}$ are the permittivity and conductivity tensor, respectively.

\subsubsection{The EQS Simulation Model}

There are different approaches for the numerical solution of electromagnetic field problems, e.g. Boundary Element Methods (BEM), Finite Elements Methods (FEM) or Finite Difference Methods (FD). The Finite Integration Technique (FIT) by Weiland (1977), see also Weiland (1996) is used throughout this paper. Like FEM, FIT is a volume oriented method, i.e. the whole space in which the solutions shall be computed is covered by small finite volumes like tetrahedron or cuboid. In contrary, BEM is surface oriented, i.e. only the surface of the solution space is discretized. All of these methods have their pros and cons. An important characteristic of FIT is that it consistently transfers Maxwell's equations into linear operator equations on the grid. Here, consistent means that all vectoranalytical and physical properties of the fields still hold on the grid. Thus, energy conservation and main other properties are ensured for the discrete solutions.

Therefore, for the numerical solution of the differential equation (10) the Finite Integration Technique (FIT) was used. Within FIT discrete linear operators replace the vectoranalytical curl-, divergence- and gradient operators. In order to setup the discrete analogue of (10) the discrete divergence operator $\widetilde{\mathbf{S}}$ and the discrete gradient operator $\mathbf{G}=-\widetilde{\mathbf{S}}^{T}$ are needed together with the discrete material operators $\mathbf{M}_{\epsilon}$ and $\mathbf{M}_{\sigma}$ and the vector $\Phi(t)$ which holds the discrete potential values in the grid points (van Rienen and Motrescu, 2003). Then, the discrete FIT equation for the transient computation of the discrete EQS potential $\Phi(t)$ is given by

$\widetilde{\mathbf{S}} \mathbf{M}_{\epsilon} \widetilde{\mathbf{S}}^{T} \frac{d}{d t} \Phi(t)+\widetilde{\mathbf{S}} \mathbf{M}_{\sigma} \widetilde{\mathbf{S}}^{T} \Phi(t)=\widetilde{\mathbf{S}} \widehat{\overline{\mathbf{j}}}(t)$,

with the discrete current flux vector $\widehat{\mathbf{j}}(t)$. Please note that in general $\mathbf{M}_{\epsilon}$ and $\mathbf{M}_{\sigma}$ are determined by the permittivity $\underline{\varepsilon}$ and conductivity $\underline{\sigma}$, i.e. non-linear, time-dependent rank two tensors which are assumed to be piecewise constant functions with $\underline{\varepsilon}>0$ and $\underline{\sigma} \geq 0$ if not stated differently.

The coupling of the discrete EQS Eq. (11) with the Hodgkin-Huxley equations is realized through the discrete current flux vector $\widehat{\mathbf{j}}(t)$ :

$\widetilde{\mathbf{S}} \mathbf{M}_{\epsilon} \widetilde{\mathbf{S}}^{T} \frac{d}{d t} \Phi(t)=\widetilde{\mathbf{S}} \widehat{\mathbf{j}}_{m}(t)$,

where $\widehat{\mathbf{j}}_{m}(t)$ holds the time-dependent sodium-, potassiumand leakage currents through the membrane. These currents are determined via the Hodgkin-Huxley equations. The discrete EQS equation (12) is then also discretized in time using a time-stepping scheme.

\subsection{Action potentials at neurons on a neurochip}

The long-term goal is to enable fully 3-D simulations of neuronal networks on sensorchips as shown e.g. in Fig. 5. For that, a numerical tool called NERVLAB has been developed 


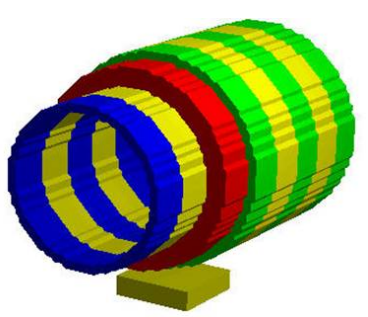

Fig. 10. Example of a simple model of an axon close to an cuboid electrode. The axon consists out of three layers, being composed of compartments. A floating potential is allocated on the electrode.

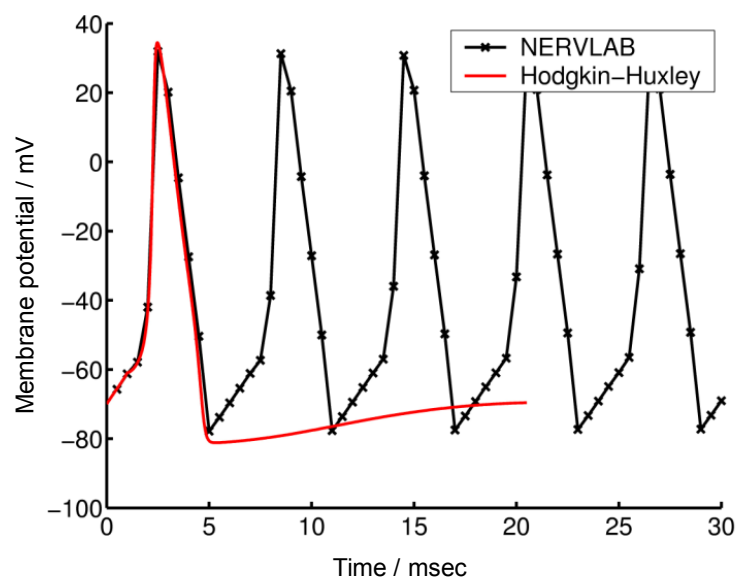

Fig. 11. Series of action potentials computed with the code NERVLAB compared with a single action potential as described by the Hodgkin-Huxley equations.

by Flehr (work in progress). This program is based on the coupling of Maxwell's equations in EQS approximation to the Hodgkin-Huxley equations where the FIT formulation (12) is used for the EQS equation. Neurons, electrode, etc. are approximated on the grid in full 3-D. Thus the neuron could have any form (round, flat in lower part but round in upper part, etc.). Of course, also the electrode and the chip can be modelled in great detail. In particular, the electrode shape could be improved under certain aspects with that tool. Also systematic studies on size and arrangement of the electrodes are made possible by NERVLAB.

The model simulates the signal transfer in a given neuron. While the Hodgkin-Huxley equations provide information only about the potential difference across the neuron's membrane, the 3-D model considers also the intra- and extracellular space with a great freedom to choose the materials outside the neuron. Thus a rather realistic model of a neurochip is possible with grids of several million nodes. For the complex situation of a full neurochip with extended neuronal network further extensions of the program are planned as e.g. domain decomposition or parallelization.

\section{Electrodes for deep brain stimulation (DBS)}

\subsection{General remarks}

DBS may be part of the advent of electric brain stimulation methods that are considered as possible treatment for a number of neurological diseases such as Parkinson's disease and other movement disorders but also cluster headaches. Before DBS became a therapy for Parkinson's disease, the electric stimulation of basal ganglia had been used to guide neurosurgeons to the precise position for a surgical lesion, the ultimate treatment of Parkinson's symptoms. The discovery of the symptomatic relief by electric stimulation led to the development of DBS as a reversible alternative to surgical lesions (Benabid and de Rougemont, 1987). For this history, DBS was not preceded by extensive work in animal models. This might also be a reason for a lack of standardization in stimulation protocols and equipment in rodent models. As can be shown, also from numeric simulations, simple downsizing of the human electrodes while maintaining stimulation protocols is not an option. Knowing this, researchers usually apply constant-current stimulation in animals as opposed to constant-voltage stimulation in humans to have varying control on the tissue field, despite of electrode geometries. However, we could show that this approach is insufficient for comparing results of different groups obtained with electrodes of different geometries. The reason is the strong geometry dependence of the tissue fields. The basis for comparing different electrode geometries must be their impedances, i.e. current-voltage relations. The electrode impedance is determined by a cell constant, i.e. a geometry factor, as well as by the specific conductivity of the surrounding medium.

\subsection{Simulation model}

For simulations, electrodes were described by threedimensional models which were immersed in a homogenous medium. Metallic parts were described as equipotential surfaces, the insulating epoxy of the electrodes and the aqueous electrolyte solution by their conductivities $(1012 \mathrm{~S} / \mathrm{m}$, $1.5 \mathrm{~S} / \mathrm{m})$ and relative permittivities $(4,80)$, respectively. The number of grid nodes equaling the number of equations has been varied from 600,000 to 1000000 . The surrounding medium was assumed to be homogeneous, isotropic and possessing aqueous properties. Nevertheless, the assumption of modified properties would only introduce minor changes in the obtained distributions.

The criterion (8) is fulfilled and the time-harmonic EQS model is appropriate. In full analogy to Eq. (9) a scalar potential is used to describe the electric field. Yet, as usual for time-harmonic field quantities, the equations are derived for the complex amplitude rather than for the field itself:

$\mathbf{E}(\mathbf{r}, t) \rightarrow \operatorname{Re}\left(\underline{\mathbf{E}}(\mathbf{r}) e^{i \omega t}\right)$

with the complex amplitude $\underline{\mathbf{E}}(\mathbf{r})=\mathbf{E}(\mathbf{r}) e^{i \phi}$ where $\phi$ is the phase angle of the cosine function. 
A derivation in analogy to that of Eq. (10) yields

$\Delta[i \omega \underline{\underline{\varepsilon}} \underline{\varphi}(\mathbf{r})+\underline{\underline{\sigma}} \underline{\varphi}(\mathbf{r})]=\operatorname{div} \underline{\mathbf{J}}_{E}(\mathbf{r})$.

Hence, a complex Poisson equation, i.e. an elliptic partial differential equation, has to be solved in order to compute the phasor $\varphi(\mathbf{r})$ of the time-harmonic EQS potential.

Again, FIT is used for the discretization leading to the governing equations

$$
\begin{aligned}
\left(i \omega \widetilde{\mathbf{S}} \mathbf{M}_{\epsilon} \widetilde{\mathbf{S}}^{T}+\widetilde{\mathbf{S}} \mathbf{M}_{\sigma} \widetilde{\mathbf{S}}^{T}\right) \Phi & =\widetilde{\mathbf{S}}\left(\mathbf{M}_{\sigma}+i \omega \mathbf{M}_{\epsilon}\right) \widetilde{\mathbf{S}}^{T} \underline{\Phi} \\
& =\widetilde{\mathbf{S}} \widehat{\widehat{\mathbf{j}}} .
\end{aligned}
$$

The linear system $\widetilde{\mathbf{S}}\left(\mathbf{M}_{\sigma}+i \omega \mathbf{M}_{\epsilon}\right) \widetilde{\mathbf{S}}^{T} \underline{\Phi}=\widetilde{\mathbf{S}} \widehat{\mathbf{j}}$ has to be solved in order to determine the EQS potential $\Phi$ with the symmetric material matrices $\mathbf{M}_{\sigma}$ and $\mathbf{M}_{\epsilon}$. Obviously, the system matrix is complex symmetric. In the past, extensive numerical studies have been carried out to find most robust and fast solution methods leading to the choice of the Krylovsubspace method BiCGCR with algebraic multigrid (AMG) as preconditioner, see e.g. (van Rienen and Weiland, 1996), (van Rienen, 2000) and (Reitzinger and van Rienen, 2003) and references therein for more details.

\subsection{Electrode processes}

In animal experiments, electrodes are usually driven in a constant-current mode to induce a temporal field behavior identical to that of the applied current. For a linear currentvoltage relation of the stimulated tissue this mode will lead to a compensation of the overpotentials by an increased electrode voltage to allow a constant current to flow. Accordingly, the temporal tissue field function should be determined by the current function. Nevertheless, the local overpotentials depend on the local current densities at the different sites of the electrode surface. When the induced tissue field is considered, electrochemical electrode properties and effects of electrode processes cannot be neglected. The electrochemical properties are determined by electrode material, medium properties and stimulation parameters. Electrochemical processes may also induce a drift in the electrode properties within minutes and up to days that is accompanied by the adsorption of organic molecules as well as metal corrosion and erosion of the plastic insulation (see Fig. 12).

Generally, an electrode current causes a potential difference at both sides of the metal-medium interface, the socalled overpotential. Overpotentials are caused by electrochemical reactions (Vetter, 1961; Gimsa and Gimsa, 2004). The intensity of electrode processes as well as the degradation of electrode metal and plastic insulation can be correlated to numerical results on the local current density at the electrode surfaces (see Fig. 12).

3.4 Induced field and electrode geometry factor (cell constant)

For numerical field simulations in the brain tissue overpotentials have been neglected. The spatial field distribution is

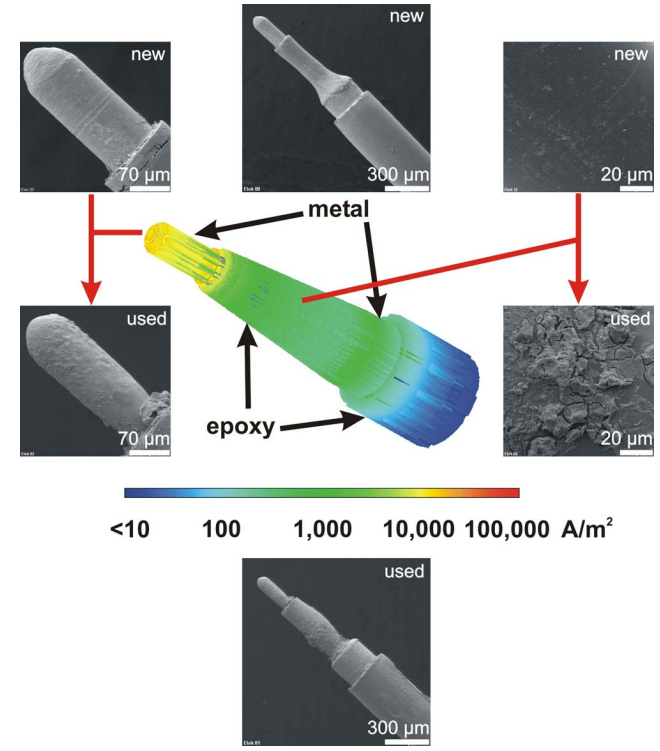

Fig. 12. Spatial distribution of the current density at the surface of a commonly used stainless steel electrode in aqueous solution of $1.5 \mathrm{~S} / \mathrm{m}$ at $1 \mathrm{~V}$ and corresponding scanning electron microscopy images before and after about $8 \mathrm{~h}$ of use. The strongest metal corrosion has been observed at the electrode tip and the interface between inner pole and insulation. The strongest erosion of the epoxy insulation occurred at the interface between insulation and outer pole.

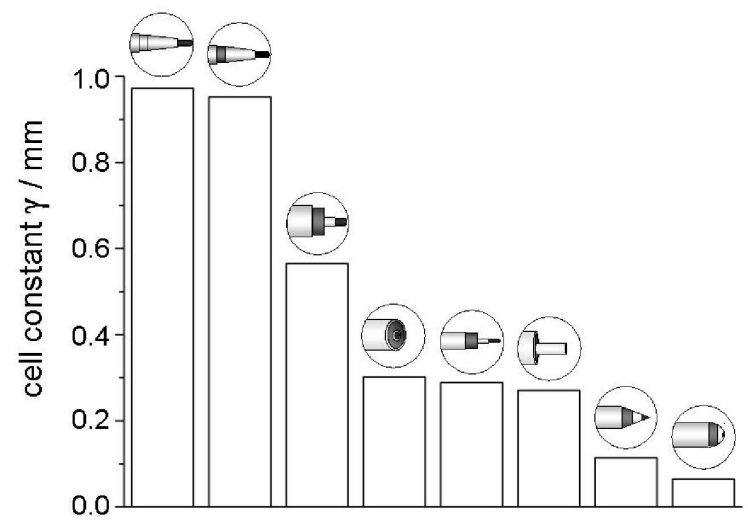

Fig. 13. Cell constants linking electrode voltage and current calculated for different electrode designs (see inserts above columns).

most important for DBS effects. To consider the affected tissue volume and the homogeneity of the field distribution, the potential, electric field and current density distributions were calculated at the electrode's metallic and insulating surfaces and in the surrounding medium, i.e. the brain tissue. In a first calculation, for each electrode geometry potentials have been set to $1 \mathrm{~V}$ and $0 \mathrm{~V}$ (inner and outer poles of the electrode, respectively). Assuming the system to be linear and homogeneous also allows for establishing proportionality factors for the current-voltage relations, i.e. the electrode-cell constants shown in Fig. 13 in combination with the conductivities of 
the surrounding media. The cell constants allow for a direct calculation of the electrode voltage corresponding to a certain drive current (e.g. of the electrode voltage at a constant current of $500 \mu \mathrm{A}$, such as often applied in animal models). The cell constants increase or decrease with a characteristic dimension for electrodes of the same shape.

\subsection{Open problems}

An additional problem is electrode ageing which is especially severe for stainless steel electrodes as used by some groups for DBS animal models (see references in Gimsa and Gimsa (2004)). At the electrode surface, the field is correlated to the current density corresponding to the intensity of electrochemical processes. These processes may drive metal corrosion and plastics erosion leading to the deposition of putative neurotoxic compounds in the brain tissue as shown for a stainless steel electrode here. However, even when noble metals and inert insulation materials are used, electrochemical processes occur which may result e.g. in gas production. Therefore, tips and edges, i.e. high curvatures, should be avoided: here the highest current densities and field strengths are located. They also set another limitation for the maximum applicable current or voltage, namely that of electric tissue damage when the induced transmembrane potential exceeds $1 \mathrm{~V}$. In turn, the maximum size of the stimulated tissue is limited for a given electrode geometry. The shape of the stimulated tissue strongly depends on the geometry of the poles of the electrode, i.e. their size and position relative to each other.

\section{Cochlear implants}

\subsection{General information}

The cochlea transforms sound-induced vibrations of the middle ear into electrical signals in the auditory nerve. The human cochlea is a tube, about $35 \mathrm{~mm}$ long, divided longitudinally into three compartments and twisted into a spiral (see Fig. 14). The three compartments are: scala vestibuli, scala tympani, and scala media which wind around the spiral preserving their spatial orientation. Reissner's membrane separates scala vestibuli from scala media, which in turn is separated from scala tympani by the spiral lamina and the basilar membrane. Scala vestibuli and scala tympani are filled with perilymph, a fluid similar to extracellular fluid, while scala media is filled with endolymph, a fluid with a high $\mathrm{K}^{+}$concentration and a low $\mathrm{Na}^{+}$concentration.

In the healthy ear, transduction of sound into electrical impulses is carried out by the organ of Corti being located on top of the basilar membrane: waves in the basilar membrane create a shear force on the hair cells, which in turn causes a change in the membrane potential of the hair cell. This is transmitted to nerve cells and from there to the brain. If the hair cells are damaged the actuation of nerve impulses is lost and thus the ability to hear. In this case a cochlear implant can help.

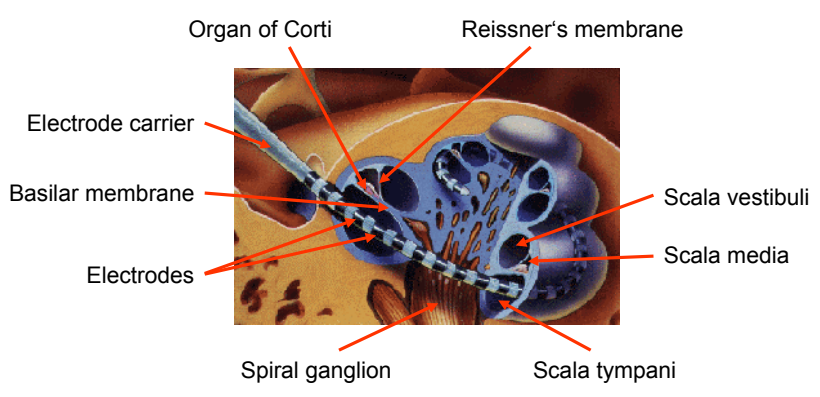

Fig. 14. Cochlea with electrode implant. Picture based on www. uni-regensburg.de/Fakultaeten/Medizin/HNO/ci/closeinner.gif.

In deaf ears with a total or subtotal loss of hair cells this mechano-acoustic converter does not function any more. In most cases however, the acoustic nerve is still in situ and working which means that it can be stimulated by electric impulses applied in its direct proximity. The idea of the socalled cochlear implant is based on this fact: an electrode array with many single electrodes is introduced into the perilymph fluid of the cochlea following the turns of the cochlear duct from the basal part till the apex. The electric impulses are generated by a microphone worn behind the patients ear, modified by a speech processor and transmitted across the skin to the electrode array implanted into the inner ear. The arrangement of the electrodes allows stimulation of nerve fibers at different levels of the cochlea representing different frequencies in the "acoustic" perception.

The stimulation of the acoustic nerve originates from a current flow between the electrodes. Three main types of stimulation are usual: monopolar, bipolar and common ground. Monopolar stimulation is based on the current flow between one electrode of the electrode carrier and a reference electrode which typically is placed outside the cochlea in the muscle tissue. In bipolar stimulation the current flow takes place between two neighboring electrodes on the carrier. Finally, a current flow between the activated electrode and all other electrodes which were switched to ground is called common ground. If the current density is high enough signals will activate the acoustic nerve fibres, action potentials will be excited and transmitted to the brain. The number of electrodes on the carrier usually varies between 15 to 24 electrodes. They are placed separately or in pairs on the last 17 to $27 \mathrm{~mm}$ of the carrier. The electrodes on the base of the cochlea actuate high frequency tones and electrodes on the top actuate low frequency tones.

There are different concepts for optimizing the "link" between the electrodes and nerve structures. One is to approach the electrodes to the "spindle" of the cochlea, the so-called modiolus, by introducing a pre-curved electrode array - a "contour advance electrode". Another concept is to insert the electrode as deep as possible into the fluid-filled cochlea. In this case the electrode is situated more laterally to the ganglion cells, but may stimulate more cells because of its long course within the cochlea. As each insertion means 
Table 1. Geometrical data of the three compartments in the unrolled cochlea model.

\begin{tabular}{lccc} 
scala & length $/ \mathrm{mm}$ & cross section $/ \mathrm{mm}^{2}$ & volume $/ \mu \mathrm{l}$ \\
\hline tympani & 26.2 & 1.22 & 31.20 \\
vestibuli & 26.2 & 0.75 & 19.64 \\
media & 26 & 0.11 & 2.76 \\
\hline
\end{tabular}

inner ear trauma, for cases with some residual hearing for high frequencies but profound hearing loss for deep tones a new method was taken into account, in which the electrode does not invade the inner ear fluids but lies in a "crevice" between the spiral ligament and the bony cochlear walls. So far, this development of an "endosteal electrode" is still in a very early stage.

Since the patients' ears vary individually, the choice of the optimal stimulation rate and pulse intensity often is difficult - especially for patients with bone diseases. In those patients it might happen that not only the acoustic nerve will be activated but also the facial nerve. This is another reason for the need of a good understanding of the electric fields and the current densities in order to allow for further improvements in cochlear implants.

\subsection{Simulation results for an unrolled model}

The field simulation is carried out with FIT using the timeharmonic equations according to Eq. (15). The geometric model considers the anatomic structures. Many of the simulation models for the cochlea with electrode implant, considering the anatomic structures, model the unrolled cochlea. One of the most elaborated winding 3-D models, to our knowledge, has been presented in Briaire and Frijns (2000a), using the boundary element method (BEM).

\subsubsection{Unrolled cochlear model}

To gain first experience the authors started off with an unrolled non-tapered model, i.e. the cross-section at the entry of the cochlea has been extended $26.2 \mathrm{~mm}$ in longitudinal direction (Fig. 15). Table 1 gives details about the compartment dimensions used in these simulations.

The largest compartment, scala tympani, is separated from the smallest compartment, scala media, by the basilar membrane. On the other side, scala media is separated from scala vestibuli by Reissner's membrane. For numerical reasons, the thickness of the membranes was enlarged to $0.05 \mathrm{~mm}$. This cochlear model is surrounded by bone as in nature the cochlea has bony walls, too. The material properties of the model are given in Table 2 .

The organ of Corti and the spiral ganglion have been neglected in this first model due to their small size and their anisotropy.

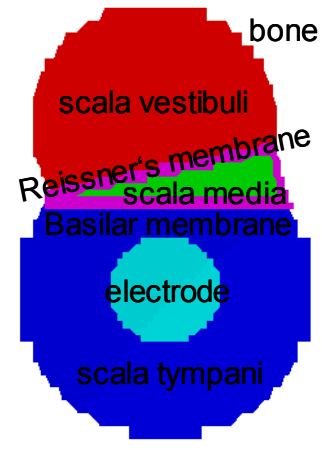

Fig. 15. Cross-section of the unrolled cochlear model. Using CFIT, the boundary approximation is much better than it appears to be from the plot.

Table 2. Material data of the three compartments of the cochlear model.

\begin{tabular}{ccc} 
material & conductivity / $(1 / \Omega \mathrm{m})$ & relative permittivity \\
\hline electrode carrier & 0 & 1 \\
perilymph & 2 & 109 \\
endolymph & 2 & 109 \\
bone & 0.083 & 1258 \\
membrane & $1.65 \cdot 10^{-3}$ & 1 \\
electrodes & $9.48 \cdot 10^{6}$ & 1 \\
& \multicolumn{2}{c}{ given potential } \\
\hline
\end{tabular}

\subsubsection{Electrodes}

In praxi, many different electrode types are used for the implementation in the cochlea. They vary in size, number of electrodes and their placement on the carrier. Here, three typical electrode carriers with their electrodes were chosen. The first model is based on the technical data from the Nucleus $24 \mathrm{k}$ (Coc) which consists of 22 ring-shaped platinum electrodes distributed in an area of $17 \mathrm{~mm}$ length along the electrode carrier. As diameter of the electrodes, $0.5 \mathrm{~mm}$ have been assumed in the model.

For the second model, the cochlear implant CLARION $\AA$ (Adv), the data of the electrode carrier is provided. Here, 16 hemispherical electrodes were placed in pairs on the carrier, which has a diameter of $0.5 \mathrm{~mm}$. The electrodes' diameter is $0.3 \mathrm{~mm}$.

The third model is based on CII Bionic Ear System ${ }^{\mathrm{TM}}$ (Adv) where 16 electrodes are placed separately on the carrier. Again, the carrier has a diameter of $0.5 \mathrm{~mm}$. The electrodes were modelled as cuboid. Some dielectric insulator is situated between the electrodes which shall minimize the interfering currents between neighboring electrodes and focus the electromagnetic field onto the acoustic nerve.

As reference electrode for the monopolar stimulation type, a spherical electrode with a diameter of $1.5 \mathrm{~mm}$ was modelled. This electrode was placed outside the cochlea. 


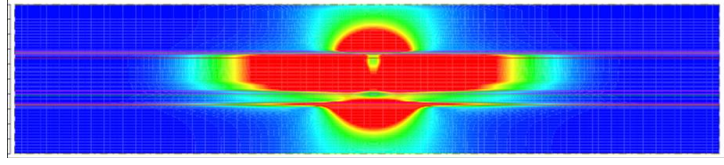

Fig. 16. Current density distribution for the Nucleus $24 \mathrm{k}$ (Coc) model with 22 ring-shaped platinum electrodes. Color plot of absolute value $|\mathbf{J}|$ over a length of $1.4 \mathrm{~cm}$.

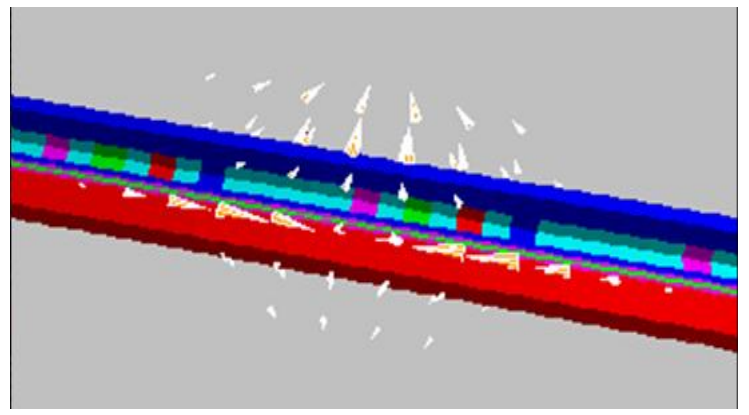

Fig. 17. Distribution of the current density $\mathbf{J}$ for the Nucleus $24 \mathrm{k}$ (Coc) model with 22 ring-shaped platinum electrodes. 3-D arrow plot for detail with 13 electrodes.

\subsubsection{Simulation of the electromagnetic fields}

First calculations were done with MAFIA 4 (CST) which is based on the Finite Integration Technique (Weiland, 1996). Static electric field and the current density were computed by Schulze (2003). Figures 16 and 17 show some recent EQS simulations by Schreiber with open boundary conditions.

A preliminary result of the calculations presented in Schulze (2003) is the guess that the bipolar stimulation type leads to the smallest scattering of the current density. For a given voltage on the electrodes, the highest chance to generate a nerve impulse is by the common ground stimulation type. Furthermore, for contour advance electrodes it could be observed that the best place for stimulation is inside the scala tympani close to the side of the acoustic nerve.

\subsection{Future aspects}

These results have to be examined with a more realistic model of cochlea and electrodes. One possibility is to setup a model of 3-D data achieved from cuts through a preparation. A CAD model for the bony walls obtained from such preparation cuts with a posteriori added endosteal electrode carrier is shown in Fig. 18. Such CAD model can be read by the program CST EMStudio ${ }^{\mathrm{TM}}$ (CST) and then be transferred to an extension of MAFIA 4 which allows for the CFIT extension of the classical Finite Integration Technique (Schreiber and van Rienen, 2004) in order to do the time-harmonic EQS simulation. If also anisotropies shall be taken into account the code SAFIT presented by Motrescu and van Rienen (2005) will be used. Numerical simulations for comparison of the

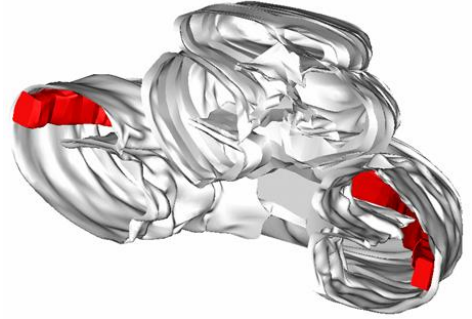

Fig. 18. CAD model for the bony cochlear walls obtained from preparation cuts with a posteriori added endosteal electrode (red).

contour advance electrode with the endosteal electrode with respect to the current spread in modiolus direction and in apical direction, respectively, as well as to side effects in the direction of nervus facialis and the middle ear are foreseen.

Another possibility is just under study (Veerathu, work in progress) where the Boundary Element Method (BEM) model of Frijns and Schoonhoven (1995), later extended by Briaire and Frijns (2000a) (see also (Briaire and Frijns, 2000b)) is read into CST EMStudio ${ }^{\mathrm{TM}}$ and transferred again to the CFIT-extension of MAFIA 4 (Schreiber and van Rienen, 2004). The advantage of this procedure is to be able to use a well established 3-D BEM model and combine it later also with a FIT-based EQS code which allows for a volume conductor model with anisotropic material (Motrescu and van Rienen, 2005). This is of importance in order to take the anisotropy of the bones around the cochlea into account. In the long run it would be nice to make individual simulations for every patient based on pictures which are made with computer tomography. Then, an optimal implant choice for every patient would be possible.

\section{Summary and outlook}

Some exemplary applications from bio-systems engineering and medical engineering have been studied in this paper. In all of the examples the EQS model was the appropriate mathematical model allowing to determine the electric field via a scalar potential. The examples originated from cooperations of the principal author recently started within Rostock University. Having applied the EQS model previously to more generic electrical engineering tasks, the author started with rather simple models to gain first experience with the novel applications. Nevertheless the studies described here sketch out well enough a rather young, but fascinating research field for electrical engineers. The principal author is convinced that detailed numerical models for this interdisciplinary field of bio-systems engineering and medical engineering will gain more and more relevance in the near future. Hereafter it is foreseen that the authors continue their joint work in order to refine and validate the models which hopefully will also help to gain an even better understanding e.g. of the causal relationship between the processes on a neurochip and the signals gained. The second major goal is 
to use the numerical models in order to improve implants in general but also, as a long-term goal, to adapt implants to the individual patient.

Acknowledgements. These studies have been supported by different grants. The work on the neurochip was supported by the European Community (EFRE) and the State Ministry of Education and Science (LFS Biosystemtechnik). The work on the mathematical model for neurochips was supported by the German Science Foundation within a post graduate programme (GRK-45). The work on the electrodes for deep brain stimulation was supported by the German Ministry of Education and Science (01ZZ0108 to U.G., StSch20020418A to J.G.). The simulation tools MAFIA 4 and CST EMStudio $^{\mathrm{TM}}$ were provided by CST GmbH, Darmstadt.

\section{References}

Advanced Bionics GmbH, Karl-Wiechert-Allee 76, D-30625 Hannover, Germany.

Baumann, W. H., Schreiber, E., Krause, G., Podssun, A., Homma, S., Schrott, R., Ehret, R., Freund, I., and Lehmann, M.: Cell monitoring system with multiparametric CMOS sensorchips, in: Proceedings $\mu$-TAS, vol. II, 554-556, Malmö, 2004.

Benabid, A. L., Pollak, P., Louveau, A., Henry, S., and de Rougemont, J.: Combined (thalamotomy and stimulation) Stereotactic Surgery of the VIM Thalamic Nucleus for Bilateral Parkinson Disease, Appl. Neurophysiol., 50, 344-346, 1987.

Bionas GmbH, Friedrich-Barnewitz-Str. 3, D-18119 Rostock, Germany.

Briaire, J. J. and Frijns, J. H. M.: 3D mesh generation to solve the electrical volume conduction problem in the implanted inner ear, Simulat. Pract. Theory, 8, 57-73, 2000a.

Briaire, J. J. and Frijns, J. H. M.: Field Patterns in a 3D Tapered Spiral Model of the Electrically Stimulated Cochlea, Hear. Res., 148, 18-30, 2000b.

Cochlear GmbH, Karl-Wiechert-Allee 76 A, D-30625 Hannover, Germany.

CST GmbH, Bad Nauheimer Str. 19, D-64289 Darmstadt, Germany.

Dirks, H. K.: Quasi-Stationary Fields for Microelectronic Applications, Electr. Eng., 79, 145-155, 1996.

Flehr, J.: Potentialberechnung zum Mechanismus der Ankopplung der Aktionspotentiale der Nervenzellen an Sensorelektroden, $\mathrm{Ph} . \mathrm{D}$. thesis, Rostock University, work in progress.

Frijns, J. H. M., de Snoo, S. L., and Schoonhoven, R.: Potential Distribution and Neural Excitation Patterns in Rotationally Symmetric Model of the Electrically Stimulated Cochlea, Hear. Res., 87, 170-186, 1995.
Gimsa, J., Habel, B., Schreiber, U., van Rienen, U., and Gimsa, U.: Choosing Electrodes for Deep Brain Stimulation Experiments Electrochemical Considerations, J. Neurosci. Meth., 142/2, 251256, 2004.

Haus, H. A. and Melcher, J. R.: Electromagnetic Fields and Energy, Prentice-Hall. Inc., 1989.

Hodgkin, A. L. and Huxley, A. F.: A quantitative description of membrane current and its application to conduction and excitation in nerve, J. Physiology, 117, 500-544, 1952.

Keener, J. and Sneyd, J.: Mathematical Physiology, Springer, 2 edn., 2001.

Koch, C.: Biophysics of Computation: Information Processing in Single Neurons, Oxford University Press, New York, 1999.

Motrescu, V. C. and van Rienen, U.: Computation of ELF electric fields in anisotropic human tissues using the Finite Integration Technique (FIT), Adv. Radio Sci., this issue (B/K), 2005.

Ransom, B. R., Neale, E., Henkart, M., Bullock, P. N., and Nelson, P. G.: Mouse spinal cord in cell culture. I, Morphology and intrinsic neuronal electrophysiologic properties, J. Neurophysiol., 40, 1132- 1150, 1977.

Reitzinger, S., Schreiber, U., and van Rienen, U.: Algebraic multigrid for complex symmetric matrices and applications, J. Computat. Appl. Math., 155, 405-421, 2003.

Schreiber, U., Clemens, M. and van Rienen, U.: Conformal FIT formulation for simulations of electro-quasistatic fields, Int. J. Appl. Electrom., 19, 193-197, 2004.

Schulze, S.: Modellierung der elektromagnetischen Felder im Umfeld eines Cochlea-Implantates, Master's thesis, Rostock University, 2003.

van Rienen, U., Clemens, M., and Weiland, T.: Simulation of LowFrequency Fields on High-Voltage Insulators with Light Contaminations, IEEE T. Mag., 32, 816-819, 1996.

van Rienen, U., Flehr, J., Schreiber, U., and Motrescu, V.: Modeling, Simulation and Optimization of Integrated Circuits, vol. 146 of International Series of Numerical Mathematics, chap. Modeling and Simulation of Electro-Quasistatic Fields, Birkhäuser Verlag, Basel/Switzerland, 17-31, 2003.

van Rienen, U.: Numerical Methods in Computational Electrodynamics - Linear Systems in Practical Applications, vol. 12 of LNCSE, Springer, 2000.

Veerathu, S.: A Contribution to Couple Boundary Element Models with Finite Integration Technique, Master's thesis, Rostock University, 2005.

Vetter, K. J.: Elektrochemische Kinetik, Springer Verlag, Berlin, Göttingen, Heidelberg, 1961.

Weiland, T.: Eine Methode zur Lösung der Maxwellschen Gleichungen für sechskomponentige Felder auf diskreter Basis, AEÜ, 31, 116-120, 1977.

Weiland, T.: Time Domain Electromagnetic Field Computation with Finite Difference Methods, JNM (Wiley), 9, 295-319, 1996.

www.uni-regensburg.de/Fakultaeten/Medizin/HNO/ci/closeinner. gif. 